\title{
Projection Versus Prewhitening for EEG Interference Suppression
}

\author{
Shun Chi Wu*, Student Member, IEEE, A. Lee Swindlehurst, Fellow, IEEE, Po T. Wang, Student Member, IEEE, \\ and Zoran Nenadic, Member, IEEE
}

\begin{abstract}
Suppression of strong, spatially correlated background interference is a challenge associated with electroencephalography (EEG) source localization problems. The most common way of dealing with such interference is through the use of a prewhitening transformation based on an estimate of the covariance of the interference plus noise. This approach is based on strong assumptions regarding temporal stationarity of the data, which do not commonly hold in EEG applications. In addition, prewhitening cannot typically be implemented directly due to ill conditioning of the covariance matrix, and $a d$ hoc regularization is often necessary. Using both simulation examples and experiments involving real EEG data with auditory evoked responses, we demonstrate that a straightforward interference projection method is significantly more robust than prewhitening for EEG source localization.
\end{abstract}

Index Terms-Electroencephalography (EEG), interference suppression, magnetoencephalography (MEG), sensor array processing, source localization.

\section{INTRODUCTION}

$\mathbf{E}$ LECTROENCEPHALOGRAPHY (EEG) and magnetoencephalography (MEG) are widely used in both clinical practice and research since these techniques provide direct measurement of cerebral activity with much higher temporal resolution compared to other noninvasive techniques such as functional magnetic resonance imaging (fMRI). Although in general EEG/MEG techniques are reported to have limited spatial resolution, high-resolution techniques for locating sources of cerebral activity have been proposed to cope with this issue [1], [2], and continue to be studied today [3]-[8]. To obtain high spatial precision, EEG/MEG localization requires a large array of sensors or electrodes, which leads to a high-dimensional inverse problem that in general does not have a unique solution. Thus, in practice, a "forward" propagation model for the brain, skull, and scalp is adopted, and one attempts to estimate the parameters

Manuscript received October 14, 2011; revised December 27, 2011 and January 26, 2012; accepted January 27, 2012. Date of publication February 10, 2012; date of current version April 20, 2012. This work was supported in part by the National Science Foundation under Grant 1056105. Asterisk indicates corresponding author.

${ }^{*}$ S. C. Wu is with the Department of Electrical Engineering and Computer Science, University of California, Irvine, CA 92697 USA (e-mail: scwu @uci.edu).

A. L. Swindlehurst is with the Department of Electrical Engineering and Computer Science, University of California, Irvine, CA 92697 USA (e-mail: swindle@uci.edu).

P. T. Wang and Z. Nenadic are with the Department of Biomedical Engineering, University of California, Irvine, CA 92697 USA (e-mail: ptwang @ uci.edu; znenadic@uci.edu).

Color versions of one or more of the figures in this paper are available online at http://ieeexplore.ieee.org.

Digital Object Identifier 10.1109/TBME.2012.2187335 of the model corresponding to the source activity. A common approach is to model the signal source in a small region of the brain as originating from an equivalent current dipole, treating the dipole location, the orientation, and magnitude of the dipole moment as dipole parameters to be estimated [9], [10].

In the literature, MUltiple SIgnal Classification (MUSIC) [1], [11], [12], linearly constrained minimum variance (LCMV) beamforming [2] and their variants such as eigenspace beamforming [3], and FIrst priNcipal vEctorS (FINES) [4] are some of the most popular high-resolution methods for estimating these dipole parameters. Under certain assumptions, these techniques can resolve very closely spaced sources with high accuracy. However, the performance of these approaches degrades significantly when strong, spatially correlated background interference is present, a situation that is not uncommon in EEG/MEG. The presence of strong unmodeled background interference (due primarily to brain activity not related to the stimulus) can degrade the performance of the algorithms to the point that the resulting parameter estimates are virtually useless, since the signals of interest are too weak to be discerned [13].

One approach to cope with the interference problem is to simultaneously estimate the interference statistics and the noise parameters using the same data, collected over several experiments involving repeated application of the stimulus. This approach was used in [14], where the dipole moments are assumed to be linear combinations of either parametric or nonparametric basis functions, and ML estimates of the spatial covariance of the interference and the parameters of the dipoles are obtained. While this approach allows for time-varying dipole moments, it requires one to assume that the interference is temporally white. In [15], the assumption of temporally white interference for dipole moments with fixed directions was relaxed through a combination of prewhitening in both the temporal and spatial domains. However, the space-time covariance of the interference is assumed to have a special structure (i.e., the Kronecker product of separate spatial and temporal noise covariance matrices) and this covariance is assumed to be unchanged from trial to trial. The assumption of an unchanging interference covariance matrix implies a level of temporal stationarity for the biological data that may be hard to justify.

Another approach to the interference suppression problem is to collect measurement data corresponding to separate "control" and "activity" conditions. The data are then analyzed to distinguish the signals of interest from the interference for further parameter estimation [5], [6], [13], [16]. Methods based on a whitening transformation of the data are the most well-known approaches for these so-called dual-condition datasets. The idea 
is to use the control state data taken prior to the application of the stimulus to estimate the covariance matrix of the interference plus noise, which in turn is used (for example) to prewhiten the data taken after the stimulus is applied, or in other words, during the "activity state." In theory, what remains in the prewhitened activity-state data will be only the transformed signals of interest with additive spatially white noise. The drawback of this approach is the need for stationarity, both during the collection of data used to estimate the covariance matrix in the control state, and then extending into the activity state. Ultimately, if the covariance matrix of the noise and interference is different during the activity state than it was during the control state, then the prewhitening may be ineffective and significant performance degradations could result.

An alternative approach to interference reduction in EEG/MEG source localization involves the use of deterministic subspace projections. In these approaches, knowledge of differences in the spatial properties of the desired and interfering signals (obtained, for example, from geometric considerations or temporal information) is exploited to project away the subspace in which the interference lies. For example, the authors in [17] demonstrated how to construct a spatial projection that filters out interfering sources that originate outside the volume of the head. Of course, interfering sources inside the head cannot be dealt with in this way. In [18], the spatial signatures of strong interferers were determined by selecting time instants where the interference is strong, and using the received data vector at those times as subspace dimensions to be projected out. However, this approach ignores information from the interferers that could be obtained at other time samples. A similar idea was applied in [19] to fetal magnetocardiography (fMCG). Since the fMCG signal is periodic with short duration, one can isolate segments of the received data where the mother's heart signal is present but not that of the fetus. The spatial signature of the mother's fMCG signal can then be projected out to reveal the weaker fetal signal.

In this paper, we use the subspace projection idea for background interference suppression in EEG/MEG source localization assuming that dual-condition datasets are available. Spatial information obtained during the control state measurements is used to find an interference subspace, and a projection matrix orthogonal to this subspace is then applied to the activity state data. This approach is more general than those mentioned earlier since the projection does not rely on any temporal properties of the interference or on prior assumptions about the locations of the interference. Unlike prewhitening, as long as the spatial locations of the interference do not significantly change between the control and activity states, the projection will be able to null the interfering sources even if their temporal structure or correlation has changed.

Our approach is a special case of a more general autoregressive filtering technique presented in [20] for a radar application, since it relies only on the stationarity of the spatial parameters of the background interference. The approach also generalizes the subspace projection technique used in [16] for the paired recursively applied and projected (RAP)-MUSIC algorithm, unlike [16], our method requires only a single interference projection, and it can be applied to essentially any source localization algorithm. Using both simulations and real data from several auditory experiments, we will demonstrate that the projection-based approach is superior to methods based on prewhitening, especially in situations where the signal-tointerference ratio (SIR) is low. Moreover, we also study the robustness of this method to two types of violations of the prerequisites for prewhitening discussed in [21]. Preliminary results of this study were presented previously in [22].

In the next section, we present the data model and assumptions made in this study. Section III reviews the use of prewhitening for standard localization techniques such as MUSIC and LCMV. The proposed subspace-based interference suppression method is then discussed in Section IV. The simulation results and real-data experiments are described in Sections V and VI, respectively. Some conclusions are offered in Section VII.

\section{StAndARD DATA MODEL AND ASSUMPTIONS}

\section{A. Current Dipole Data Model}

Following the mathematical model of [1], [2], [13], and [14], the outputs of $m$ sensors sampled at some time instant $t$ are stacked in an $m \times 1$ vector $\mathbf{x}(t)$. The sensor array can be composed of either electrodes as with EEG devices, detector coils as in MEG systems, or both. In this paper, we will focus on the EEG application, although from a mathematical standpoint, the techniques we develop are applicable to MEG localization as well. If $N_{S}$ dipoles are present in response to a given stimulus, the output of the array may be modeled as a linear superposition of the signals from each source:

$$
\mathbf{x}(t)=\mathbf{A s}(t)+\mathbf{n}(t)
$$

where the $N_{S} \times 1$ signal vector $\mathbf{s}(t)$ is composed of the moment magnitudes of the $N_{S}$ dipoles sampled at time $t, \mathbf{n}(t) \in \mathbb{R}^{m \times 1}$ is composed of both background electronic/sensor noise and contributions from interfering EEG sources that are nonstimulusrelated and, therefore, not of interest. We will let $N_{I}$ represent the number of significant interfering dipoles that contribute to $\mathbf{n}(t)$. The matrix $\mathbf{A}=\left[\mathbf{a}_{1}, \mathbf{a}_{2}, \ldots, \mathbf{a}_{N_{S}}\right]$ is comprised of $N_{S}$ vectors of dimension $m \times 1$ that correspond to the response of the array to each dipole source. These response vectors are often referred to as lead field vectors (LFV). The $k$ th LFV $\mathbf{a}_{k}\left(\mathbf{r}_{k}, \phi_{k}\right)$ represents the response of the sensor array to a unit-magnitude dipole located at position $\mathbf{r}_{k}$ with dipole orientation denoted by

$$
\phi_{k}=\left[\begin{array}{lll}
\phi_{x k} & \phi_{y k} & \phi_{z k}
\end{array}\right]^{T} .
$$

In practice, a current dipole is a good approximation for a small source viewed from a remote field point [10] and its corresponding LFV can be derived from multilayer spherical head models [1], [2], [9], [13], [14] or based on realistic head measurements taken from computed tomography or MRI data [23].

The LFV model is typically decomposed into three parts, each due to a separate orientation component:

$$
\begin{aligned}
\mathbf{a}_{k}\left(\mathbf{r}_{k}, \phi_{k}\right) & =\mathbf{G}\left(\mathbf{r}_{k}\right) \phi_{k} \\
& =\left[\begin{array}{lll}
\mathbf{g}_{x}\left(\mathbf{r}_{k}\right) & \mathbf{g}_{y}\left(\mathbf{r}_{k}\right) & \mathbf{g}_{z}\left(\mathbf{r}_{k}\right)
\end{array}\right] \phi_{k}
\end{aligned}
$$

for $k=1, \ldots, N_{S}$, where $\mathbf{g}_{x}\left(\mathbf{r}_{k}\right), \mathbf{g}_{y}\left(\mathbf{r}_{k}\right)$, and $\mathbf{g}_{z}\left(\mathbf{r}_{k}\right)$ are the $m \times 1$ LFV's that result from unit magnitude dipole sources 
oriented in the $x$-, $y$ - and $z$-directions, respectively. Note also that there is an inherent scaling ambiguity in (1) between each element $s_{k}(t)$ of $\mathbf{s}(t)$ and the corresponding orientation vector $\phi_{k}$. The ambiguity is usually resolved by constraining $\phi_{k}$ in some way, and absorbing any scale factor into $s_{k}(t)$. For example, $\left\|\phi_{k}\right\|=1$ or $\phi_{x k}=1$ are common choices, each with its own implications about the moment magnitude $s_{k}(t)$ [24].

\section{B. Assumptions}

We will adopt the following assumptions regarding the data model described earlier:

A1 The total number of dipole sources (stimulus and nonstimulus related) of sufficient strength to contribute to the model is less than the total number of sensors: $N_{I}+N_{S}<m$.

A2 Any collection of $p \leq m$ LFV forms a linearly independent set (in other words, the sensor array is unambiguous).

A3 The dipole moment magnitudes are zero-mean and uncorrelated with $\mathbf{n}(t)$, and the moment magnitudes from different source dipoles are linearly independent (i.e., the moment of one source dipole cannot be expressed as a linear combination of those of other source dipoles).

A4 The location of all interfering sources (biological or otherwise) is time-invariant. In particular, interfering sources present during the control state remain in the same location during the activity state. On the other hand, the sources of interest are only assumed to be present during the activity state, after the stimulus is applied, and not during the control state.

A5 All other components of $\mathbf{n}(t)$ (due to electronic noise, etc.) are assumed to be zero mean, spatially white with an equal variance of $\sigma^{2}$ across all sensors, and relatively weak compared to the interference.

Assumptions A2, A3, and A5 are standard for existing approaches described in the literature. Assumption A4 is implied in other existing approaches as well, but these approaches also make additional restrictive assumptions requiring white or at least stationary temporal statistics for the moment magnitudes of the interfering sources. Here, we do not require that these signals be white or even stationary. Furthermore, we will examine the impact of violations to our assumption A4 later in this paper. A1 is slightly more restrictive than the corresponding condition for methods based on prewhitening, which is $N_{I}<m$. In most applications of interest, $m$ is large enough and $N_{S}$ is small enough for A1 to still be reasonable. Note that technically, as we will see later, it is not the number of interfering sources that is important, but rather the effective rank $\rho$ of their spatial covariance. This rank can be significantly smaller than $N_{I}$ (for example, due to the presence of clusters of interfering sources), and $\mathrm{A} 1$ can actually be stated as requiring the less constraining condition $\rho+N_{S}<m$.

\section{Dual-Condition Experiments and Prewhitening}

For EEG measurements, the signals of interest are very weak, and embedded in strong, spatially correlated noise and interference due primarily to background brain activity not related to the stimulus. A common strategy in such situations is to design experiments with dual conditions, one (control state) prior to application of the stimulus and one (activity state) after the stimulus has been applied [6], [13], [16], [21]. In principle, the control state data will contain only background interference and sensor noise, while the activity state data will contain statistically similar noise and interference as well as the event-related signals. In the standard prewhitening approach, the control state data are first used to estimate the spatial covariance matrix of the interference plus noise using, for example, the following sample average:

$$
\hat{\mathbf{R}}_{C}=\frac{1}{n_{C}} \sum_{t=1}^{n_{C}} \mathbf{x}_{C}(t) \mathbf{x}_{C}^{T}(t)
$$

where $n_{C}$ is the number of control state samples and $\mathbf{x}_{C}(t)$ is assumed to be zero-mean (assumption A5). The activity state data $\mathbf{x}_{A}(t)$ are then prewhitened to eliminate the influence of the interference and noise as follows:

$$
\mathbf{x}_{A}^{\prime}(t)=\hat{\mathbf{R}}_{C}^{-1 / 2} \mathbf{x}_{A}(t) .
$$

As mentioned earlier, the use of prewhitening presumes that the spatial and temporal statistics of the interference and noise during the control state are identical to those during the activity state. As illustrated later in the simulation and experimental results, if the assumption of stationarity between these two states is violated, then methods based on prewhitening can suffer a significant performance degradation.

\section{LOCALIZATION ALGORITHMS}

We briefly review here the MUSIC and LCMV approaches for dipole localization based on prewhitening. The prewhitening approach is quite general, and can be applied to other algorithms such as maximum likelihood, eigenspace beamforming, RAP-MUSIC, FINES, etc. Furthermore, the subspace projection approach we present later can be applied in any situation where prewhitening is used. Thus, our discussion of MUSIC and LCMV in this paper is simply to provide examples of how the algorithms are implemented. If the noise and interference are spatially and temporally stationary, and one could exactly determine the covariance matrices $\mathbf{R}_{C}$ and $\mathbf{R}_{A}$ for the control and activity states, respectively, they would be related as follows:

$$
\begin{aligned}
\mathbf{R}_{A}^{\prime} & =\mathbf{R}_{C}^{-1 / 2} \mathbf{A} \mathbf{Q}_{S} \mathbf{A}^{T} \mathbf{R}_{C}^{-1 / 2}+\mathbf{I} \\
& =\sum_{i=1}^{m} \lambda_{i} \mathbf{e}_{i} \mathbf{e}_{i}^{T}=\mathbf{E}_{S}^{\prime} \Lambda_{S}^{\prime} \mathbf{E}_{S}^{\prime T}+\mathbf{E}_{N}^{\prime} \mathbf{E}_{N}^{\prime T}
\end{aligned}
$$

where $\mathbf{Q}_{S}$ is the covariance matrix of the dipole magnitudes, and the second equation describes the eigendecomposition of $\mathbf{R}_{A}^{\prime}$. The eigenvalues are ordered as $\lambda_{1} \geq \ldots \geq \lambda_{N_{S}}>\lambda_{N_{S}+1}=$ $\ldots=\lambda_{m}=1$, where the $m-N_{S}$ smallest eigenvalues are equal to one due to the prewhitening and assumption A1. The matrix $\mathbf{E}_{S}^{\prime}=\left[\mathbf{e}_{1}, \ldots, \mathbf{e}_{N_{S}}\right]$ contains the eigenvectors corresponding to the $N_{S}$ largest eigenvalues contained in the diagonal matrix $\Lambda_{S}^{\prime}$, and $\mathbf{E}_{N}^{\prime}=\left[\mathbf{e}_{N_{S}+1}, \ldots, \mathbf{e}_{m}\right]$ contains the eigenvectors with eigenvalues equal to one. We refer to $\mathbf{E}_{S}^{\prime}$ and $\mathbf{E}_{N}^{\prime}$ as the signal and noise subspace eigenvectors, respectively. The value 
of $N_{S}$ is revealed by the number of eigenvalues in $\mathbf{R}_{A}^{\prime}$ that are greater than one. The aforementioned result is independent of the presence of noise and the signal-to-noise ratio (SNR), provided that perfect measurements of $\mathbf{R}_{C}$ and $\mathbf{R}_{A}^{\prime}$ are available. However, since only a finite amount of data can be collected, the best we can do in practice is to use sample averages such as (4) to estimate $\mathbf{R}_{C}$ and $\mathbf{R}_{A}^{\prime}$ so that the $m-N_{S}$ eigenvalues will no longer be exactly equal to one. Consequently, determination of $N_{S}$ is less obvious, but still possible if the postinterference suppression $\mathrm{SNR}$ is reasonably high and $\mathbf{Q}_{S}$ is full rank as required in A3. Methods for determining $N_{S}$ in such cases have been presented in [25] and [26].

\section{A. MUltiple SIgnal Classification}

MUSIC [1], [6], [11], [12] locates the dipoles as those whose LFV's are most orthogonal to $\mathbf{E}_{N}^{\prime}$, i.e.

$$
\begin{aligned}
\hat{\mathbf{r}}, \hat{\boldsymbol{\phi}} & =\arg \min _{\mathbf{r}, \boldsymbol{\phi}} V_{\mathrm{MU}}(\mathbf{r}, \boldsymbol{\phi}) \\
& =\arg \min _{\mathbf{r}, \boldsymbol{\phi}} \frac{\phi^{T} \mathbf{G}^{\prime T}(\mathbf{r}) \mathbf{E}_{N}^{\prime} \mathbf{E}_{N}^{\prime T} \mathbf{G}^{\prime}(\mathbf{r}) \boldsymbol{\phi}}{\boldsymbol{\phi}^{T} \mathbf{G}^{\prime T}(\mathbf{r}) \mathbf{G}^{\prime}(\mathbf{r}) \boldsymbol{\phi}}
\end{aligned}
$$

where $\mathbf{r}$ is the 3-D coordinates of a possible dipole, $\phi$ is its orientation as defined in (2), and $\mathbf{G}^{\prime}(\mathbf{r})=\hat{\mathbf{R}}_{C}^{-1 / 2} \mathbf{G}(\mathbf{r})$. A natural constraint for $\phi$ in this case is $\phi^{T} \phi=1$, in which case minimizing the MUSIC criterion is equivalent to finding the minima of the following generalized eigenvalue problem:

$$
\begin{aligned}
\hat{\mathbf{r}} & =\arg \min _{\mathbf{r}} \lambda_{\min }(\mathbf{r}) \\
\mathbf{G}^{\prime T}(\hat{\mathbf{r}}) \mathbf{E}_{N}^{\prime} \mathbf{E}_{N}^{\prime T} \mathbf{G}^{\prime}(\hat{\mathbf{r}}) \hat{\boldsymbol{\phi}} & =\lambda_{\min }(\hat{\mathbf{r}}) \mathbf{G}^{\prime T}(\hat{\mathbf{r}}) \mathbf{G}^{\prime}(\hat{\mathbf{r}}) \hat{\boldsymbol{\phi}} .
\end{aligned}
$$

The position estimates are found by viewing $\lambda_{\min }$ as a function of $\mathbf{r}$ and then searching for its minima. The orientation of the dipole with location estimate $\hat{\mathbf{r}}$ is then taken to be the unit-norm eigenvector associated with $\lambda_{\min }(\hat{\mathbf{r}})$. MUSIC is referred to as a "scanning" method, since it relies on a scan of the 3-D volume in order to localize potential sources.

\section{B. Linearly Constrained Beamforming}

The LCMV approach scans by forming "beams" that minimize power subject to a unit gain constraint at each potential source location [2], [7], [13]. This leads to finding the minima of the following cost function:

$$
\begin{aligned}
\hat{\mathbf{r}}, \hat{\boldsymbol{\phi}} & =\arg \min _{\mathbf{r}, \boldsymbol{\phi}} V_{\mathrm{LCMV}}(\mathbf{r}, \boldsymbol{\phi}) \\
& =\arg \min _{\mathbf{r}, \boldsymbol{\phi}} \frac{\boldsymbol{\phi}^{T} \mathbf{G}^{\prime T}(\mathbf{r})\left(\mathbf{R}_{A}^{\prime}\right)^{-1} \mathbf{G}^{\prime}(\mathbf{r}) \boldsymbol{\phi}}{\boldsymbol{\phi}^{T} \mathbf{G}^{\prime T}(\mathbf{r}) \mathbf{G}^{\prime}(\mathbf{r}) \boldsymbol{\phi}} .
\end{aligned}
$$

The positions and orientations are then found in the same fashion as in MUSIC:

$$
\begin{aligned}
\hat{\mathbf{r}} & =\arg \min _{\mathbf{r}} \lambda_{\min }(\mathbf{r}) \\
\mathbf{G}^{\prime T}(\hat{\mathbf{r}})\left(\mathbf{R}_{A}^{\prime}\right)^{-1} \mathbf{G}^{\prime}(\hat{\mathbf{r}}) \hat{\boldsymbol{\phi}} & =\lambda_{\min }(\hat{\mathbf{r}}) \mathbf{G}^{\prime T}(\hat{\mathbf{r}}) \mathbf{G}^{\prime}(\hat{\mathbf{r}}) \hat{\boldsymbol{\phi}} .
\end{aligned}
$$

\section{SUBSPACE-BASED INTERFERENCE SUPPRESSION}

In this section, a deterministic alternative to prewhitening for interference suppression is presented. Instead of using the control state measurements to estimate the covariance matrix of the interference, the measurements are used to construct a subspace that is orthogonal to it. We break down the data collected during the control and activity states as follows:

$$
\begin{array}{ll}
\text { Control: } & \mathbf{X}_{C}=\mathbf{A}_{I} \mathbf{S}_{I C}+\mathbf{W}_{C} \\
\text { Activity: } & \mathbf{X}_{A}=\mathbf{A}_{S} \mathbf{S}_{S}+\mathbf{A}_{I} \mathbf{S}_{I A}+\mathbf{W}_{A}
\end{array}
$$

where $\mathbf{A}_{I}$ represents the LFV's of the $N_{I}$ interfering sources present during both the control and activity states, $\mathbf{S}_{I C}=$ $\left[\mathbf{s}_{I C}(1), \ldots, \mathbf{s}_{I C}\left(n_{C}\right)\right]$ contains the dipole magnitudes of the interfering sources during the control state, $\mathbf{S}_{I A}$ corresponds to the magnitudes of the interference sources during the activity state, $\mathbf{A}_{S}$ contains the LFV's of the new sources of interest that appear during the activity state, $\mathbf{S}_{S}=\left[\mathbf{s}_{S}(1), \ldots, \mathbf{s}_{S}\left(n_{A}\right)\right]$ contains the dipole magnitudes of the sources of interest, and $\mathbf{W}_{C}, \mathbf{W}_{A}$ represent the spatially white background noise present during the control and activity states, respectively. The noise terms $\mathbf{W}_{C}, \mathbf{W}_{A}$ are taken to be spatially white since we assume that any nonspatially-white components that are not stimulus-related are due to interfering sources (assumptions A4 and A5), and all interfering sources are included as terms in $\mathbf{A}_{I}$. No assumptions are made about the temporal properties of $\mathbf{W}_{C}, \mathbf{W}_{A}$; they can be temporally colored with arbitrary correlation properties. We also emphasize that we make no assumptions about the relationship between $\mathbf{S}_{I C}$ and $\mathbf{S}_{I A}$; in fact, they may be completely unrelated to each other. Furthermore, some rows of $\mathbf{S}_{I A}$ may be zero, indicating that only a portion of the interfering sources may be present during the activity state (we will discuss this latter case later in the section).

Because of the presence of $\mathbf{W}_{C}$ and $\mathbf{W}_{A}$, both $\mathbf{X}_{C}$ and $\mathbf{X}_{A}$ will in general be full rank. However, assuming that the noninterference-related background noise $\mathbf{W}_{C}, \mathbf{W}_{A}$ is relatively weak compared with $\mathbf{S}_{I C}, \mathbf{S}_{I A}, \mathbf{S}_{S}$ (assumption A5), the bulk of the energy in $\mathbf{X}_{A}$ will be confined to the $N_{S}+N_{I}$ dimensional subspace defined by $\mathcal{S}_{A}=\operatorname{span}\left(\left[\begin{array}{ll}\mathbf{A}_{S} & \mathbf{A}_{I}\end{array}\right]\right)$, while the bulk of the energy in $\mathbf{X}_{C}$ will lie in a subspace of $\mathcal{S}_{C}$ defined by $\mathcal{S}_{C}=\operatorname{span}\left(\mathbf{A}_{I}\right) \subset \mathcal{S}_{A}$. Under the aforementioned assumptions, a reasonable approach for eliminating the effect of the interference in $\mathbf{X}_{A}$ would be to project its columns onto the space orthogonal to $\mathcal{S}_{C}$ prior to localizing the sources of interest. Components of $\mathbf{A}_{S}$ nearly parallel to $\mathbf{A}_{I}$ will be lost in this process, but a similar signal cancellation effect occurs in such situations with prewhitening as well. Although the idea is quite straightforward, we will see that it provides significantly more robust performance than prewhitening methods in both simulated and real data experiments.

Mathematically, the projection operator can be found in a standard way. Following the approach of [20], we estimate an $m \times m-N_{I}$ matrix $\mathbf{H}$ orthogonal to $\mathcal{S}_{C}$ by the following leastsquares problem:

$$
\hat{\mathbf{H}}=\arg \min _{\mathbf{H}}\left\|\mathbf{H}^{T} \mathbf{X}_{C}\right\|_{F}^{2}
$$


subject to the constraint $\mathbf{H}^{T} \mathbf{H}=\mathbf{I}$ to avoid a trivial solution. The solution for $\mathbf{H}$ can be quantified in terms of the singular value decomposition (SVD) of the control state measurements, due to the assumption that the majority of the energy in $\mathbf{X}_{C}$ is confined to an $N_{I}$ dimensional subspace defined by $\mathbf{A}_{I}$. For example, assuming $n_{C} \geq m$, where $n_{C}$ is the number of control state data samples, the SVD will be given by

$$
\begin{aligned}
\mathbf{X}_{C} & =\mathbf{U}_{C} \boldsymbol{\Sigma}_{C} \mathbf{V}_{C}^{T} \\
& =\left[\begin{array}{ll}
\mathbf{U}_{I} & \mathbf{U}_{N}^{C}
\end{array}\right]\left[\begin{array}{ccc}
\boldsymbol{\Sigma}_{I} & \mathbf{0} & \mathbf{0} \\
\mathbf{0} & \boldsymbol{\Sigma}_{N}^{C} & \mathbf{0}
\end{array}\right] \mathbf{V}_{C}^{T}
\end{aligned}
$$

which leads to $\hat{\mathbf{H}}=\mathbf{U}_{N}^{C}$, where $\mathbf{U}_{N}^{C}$ is the matrix of the $m-N_{I}$ left singular vectors of $\mathbf{X}_{C}$ corresponding to the smallest singular values. The corresponding projection matrix is given by $\mathbf{P}_{H}=\hat{\mathbf{H}} \hat{\mathbf{H}}^{T}$. The same solution is obtained for the case where $m>n_{C}>N_{I}$; only the dimensions of the matrix containing the singular values changes. Projecting the activity state measurements onto the subspace defined by $\mathbf{P}_{H}$, we have

$$
\mathbf{P}_{H} \mathbf{X}_{A} \simeq \mathbf{P}_{H} \mathbf{A}_{S} \mathbf{S}_{S}+\mathbf{P}_{H} \mathbf{W}_{A}
$$

and the contribution of the interferers is approximately removed.

The interference projection alters the lead field matrix of the desired sources, so MUSIC and LCMV must be modified prior to implementation. Note that although multiplication by $\mathbf{P}_{H}$ has made the background noise term in (11) nonwhite, this does not cause a problem since $\mathbf{P}_{H}$ is an orthogonal projection, and the signal term is confined to the subspace defined by $\mathbf{P}_{H}$ as well. We simply modify the LFV's by multiplying them by $\mathbf{P}_{H}$, and otherwise proceed to implement MUSIC as normal. Define $\mathbf{X}_{A}^{\prime \prime}=\mathbf{P}_{H} \mathbf{X}_{A}$ and $\mathbf{G}^{\prime \prime}(\mathbf{r})=\mathbf{P}_{H} \mathbf{G}(\mathbf{r})$, as well as the following SVD:

$$
\begin{aligned}
\mathbf{X}_{A}^{\prime \prime} & =\mathbf{U}_{A} \boldsymbol{\Sigma}_{A} \mathbf{V}_{A}^{T} \\
& =\left[\begin{array}{ll}
\mathbf{U}_{S} & \mathbf{U}_{N}^{\prime \prime}
\end{array}\right]\left[\begin{array}{ccc}
\boldsymbol{\Sigma}_{S} & \mathbf{0} & \mathbf{0} \\
\mathbf{0} & \mathbf{\Sigma}_{N}^{\prime \prime} & \mathbf{0}
\end{array}\right] \mathbf{V}_{A}^{T}
\end{aligned}
$$

where $\mathbf{U}_{S}$ contains the $N_{S}$ left singular vectors with largest singular values. With this notation, the modified MUSIC approach becomes

$$
\begin{gathered}
\hat{\mathbf{r}}=\arg \min _{\mathbf{r}} \lambda_{\min }(\mathbf{r}) \\
\mathbf{G}^{\prime \prime T}(\hat{\mathbf{r}}) \mathbf{E}_{N}^{\prime \prime} \mathbf{E}_{N}^{\prime \prime T} \mathbf{G}^{\prime \prime}(\hat{\mathbf{r}}) \hat{\boldsymbol{\phi}}=\lambda_{\min }(\hat{\mathbf{r}}) \mathbf{G}^{\prime \prime T}(\hat{\mathbf{r}}) \mathbf{G}^{\prime \prime}(\hat{\mathbf{r}}) \hat{\boldsymbol{\phi}} .
\end{gathered}
$$

with $\mathbf{E}_{N}^{\prime \prime}=\mathbf{U}_{N}^{\prime \prime}$. We will refer to this approach as null projection (NP)-MUSIC. The NP version of LCMV can be similarly obtained using an estimate $\hat{\mathbf{R}}_{A}^{\prime \prime}$ of the covariance of $\mathbf{X}_{A}^{\prime \prime}$, as follows:

$$
\begin{gathered}
\hat{\mathbf{r}}=\arg \min _{\mathbf{r}} \lambda_{\min }(\mathbf{r}) \\
\mathbf{G}^{\prime \prime T}(\hat{\mathbf{r}})\left(\mathbf{R}_{A}^{\prime \prime}\right)^{-1} \mathbf{G}^{\prime \prime}(\hat{\mathbf{r}}) \hat{\boldsymbol{\phi}}=\lambda_{\min }(\hat{\mathbf{r}}) \mathbf{G}^{\prime \prime} T(\hat{\mathbf{r}}) \mathbf{G}^{\prime \prime}(\hat{\mathbf{r}}) \hat{\boldsymbol{\phi}} .
\end{gathered}
$$

Two types of violations of the prerequisites for prewhiteningbased interference suppression that may arise in dual conditional experiments were presented by [21]. In this section, we discuss the robustness of the NP-projection-based interference suppression approach to these two scenarios. These scenarios correspond to violations of our assumption A4. Another case involving interfering sources that change location from the control to the activity state will be examined in the simulation examples.

1) Control-only source scenario: In this case, it is assumed that there are some interference sources that exist only in the control state and do not appear in the activity state, as follows:

$$
\begin{aligned}
& \text { Control: } \mathbf{X}_{C}=\mathbf{A}_{I} \mathbf{S}_{I C}+\mathbf{A}_{\Delta} \mathbf{S}_{\Delta}+\mathbf{W}_{C} \\
& \text { Activity: } \mathbf{X}_{A}=\mathbf{A}_{S} \mathbf{S}_{S}+\mathbf{A}_{I} \mathbf{S}_{I A}+\mathbf{W}_{A} .
\end{aligned}
$$

Under this circumstance the estimated null space will be orthogonal to $\left\{\mathbf{A}_{I}, \mathbf{A}_{\Delta}\right\}$ which is the subspace spanned by the interference and the control-only sources. In this case, the NP is removing a higher dimensional space from the data than is needed to eliminate the interference corresponding to $\mathbf{A}_{I}$. This will reduce the dimensionality of the space available for locating and discriminating the dipole sources of interest, and the SNR of these signals will be degraded. The impact the control-only sources will depend on the dimension of $\mathbf{A}_{\Delta}$ and on the degree to which it overlaps with $\mathbf{A}_{S}$. As long as the number of such interferers is small and their locations are not near those of the sources of interest, the impact on NP-based methods will not be significant.

2) Modulating source scenario: In this scenario, the sources of interest are active prior to the activity state, although with a weaker signal strength than after the stimulus is applied. Mathematically, we have the following data model:

$$
\begin{aligned}
& \text { Control: } \mathbf{X}_{C}=\mathbf{A}_{S} \mathbf{S}_{S C}+\mathbf{A}_{I} \mathbf{S}_{I C}+\mathbf{W}_{C} \\
& \text { Activity: } \mathbf{X}_{A}=\mathbf{A}_{S} \mathbf{S}_{S A}+\mathbf{A}_{I} \mathbf{S}_{I A}+\mathbf{W}_{A} .
\end{aligned}
$$

Problems will arise for the NP-based methods (as well as prewhitening-based approaches) unless $\left\|\mathbf{S}_{S C}\right\| \ll\left\|\mathbf{S}_{I C}\right\|$ so that $N_{I}$ can still be reliably determined. In the simulations of Section V, we illustrate the impact of this situation on the performance of the NP and prewhitening-based algorithms.

\section{Simulation EXAmPLES}

\section{A. Simulation Parameters}

As an example, we choose an EEG simulation involving 61 electrodes from the 10-10 system [27]. The LFV's were calculated using the approximate method of [9], in which the head was modeled as a four-layer sphere with its radii and conductivities specified in [28]. The number of interfering sources was set to 25 , and were placed randomly on the surface of brain with random dipole orientations. Rather than generating them synthetically, the waveforms used for the interfering sources were taken from the electrode outputs of real EEG measurements. The number of sources of interest was 3 in the simulations, and they were also located on the brain surface with random orientation. The moment magnitudes for the sources of interest were chosen to be similar to the averaged evoked response potentials (ERP) illustrated in [29]. White noise was added to both control and activity state data with its power being specified by a given 

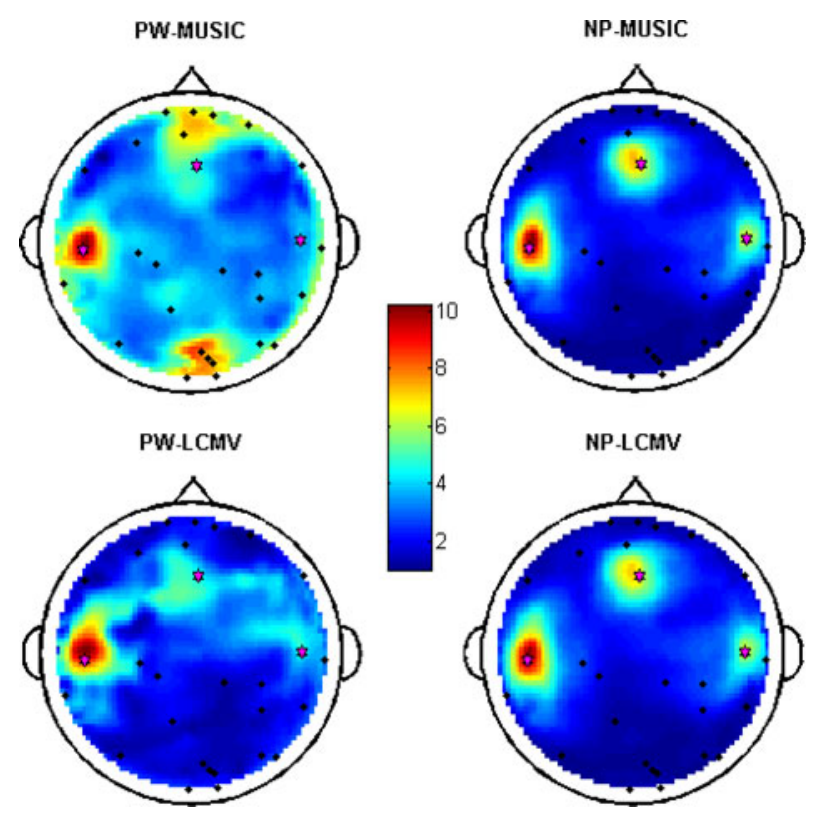

Fig. 1. Spatial spectra of the four algorithm combinations (top view). Black dots represent locations of interferers, while the magenta stars represent the true positions of the sources of interest, respectively. The SIR and SNR were respectively 0 and $15 \mathrm{~dB}$.

SNR, defined as $\left\|\mathbf{A}_{S} \mathbf{S}_{S}\right\|_{F}^{2} /\left\|\mathbf{W}_{A}\right\|_{F}^{2}$. Each signal of interest had unit power and all interference waveforms had the same power specified by the SIR $\left\|\mathbf{A}_{S} \mathbf{S}_{S}\right\|_{F}^{2} /\left\|\mathbf{A}_{I} \mathbf{S}_{I}\right\|_{F}^{2}$.

\section{B. Results and Discussion}

The first example compares the performance of the proposed interference suppression approach with prewhitening. In this example, the SNR was set at $15 \mathrm{~dB}$ and the SIR varied from 5 to $-5 \mathrm{~dB}$. Three dipole sources were placed in fixed positions on the surface of brain, near sensors Fz, C5 and C6. The dipole orientations were chosen randomly. Fig. 1 shows the spatial spectra of the four algorithm combinations, obtained by evaluating the reciprocals of (7) and (8) at the grid points (we plot the inverse of the cost functions so that the peaks in the spectrum correspond to the source locations). PW stands for "prewhitened" in the figure legend. Magenta stars represent the true positions of the sources of interest. Black dots show the locations of the interfering sources. The SIR for this example was $0 \mathrm{~dB}$. Fig. 1 illustrates that the direct interference cancellation approaches do a significantly better job of removing the effects of the background noise, while the prewhitened approaches still suffer from the influence of the interference (particularly the PW-MUSIC approach). Due to their inability to suppress the interference, the PW-based approaches may not be able to locate the sources, especially when the SIR is low (as shown in Fig. 1 by noticing the missing peaks around the magenta stars that represent the true positions of the sources of interest). In some trials, the PWbased approaches locate the interferers instead of the sources of interest. To emphasize that it is the temporal nonstationarity of the data that is causing difficulties for the prewhitening-based approaches, Fig. 2 shows the results obtained in the artificial

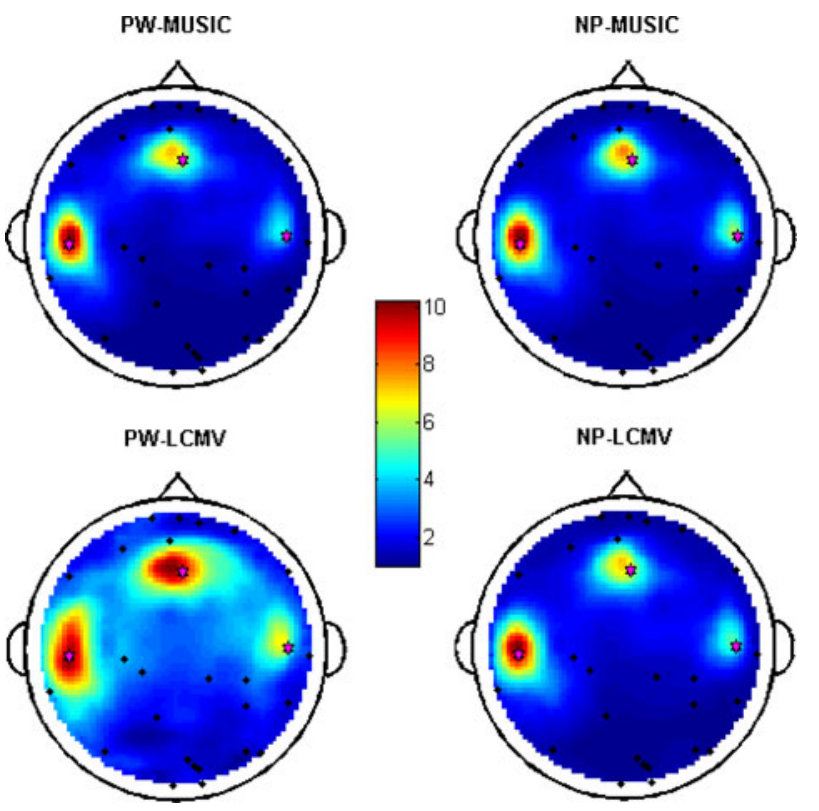

Fig. 2. Same as previous figure but the interfering signals have the same statistics in both the control and activity states.

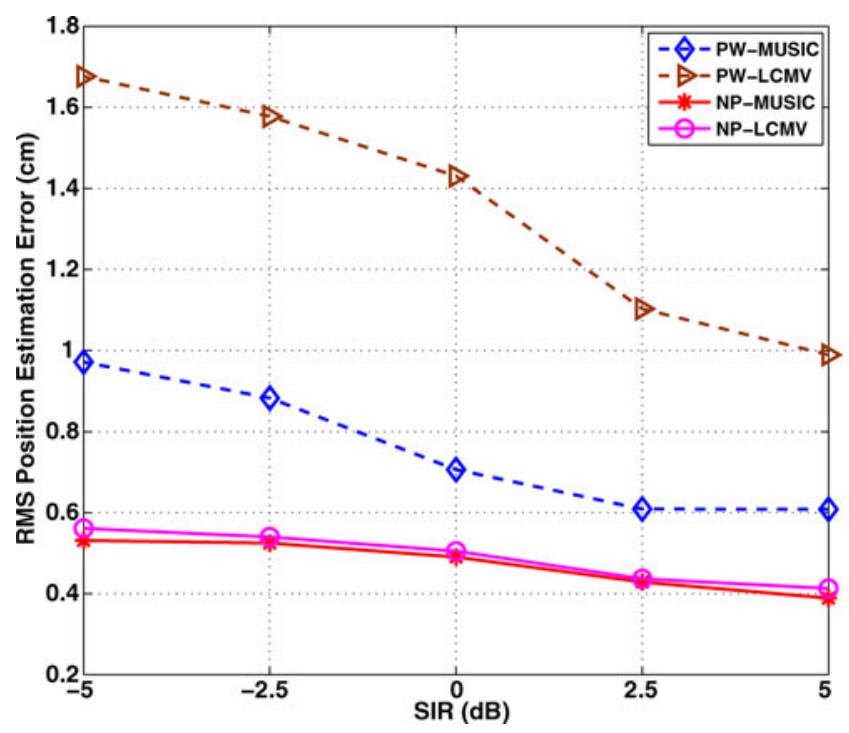

Fig. 3. RMS position estimation errors of the four algorithm combinations.

case where the dipoles produce identical signals during both the control and activity states. In this case, where temporal stationarity is artificially guaranteed, the performance of the projection and prewhitening methods is essentially the same.

To quantify the performance gain of NP, we conducted a series of Monte Carlo trials in which the sources of interest were in the same locations with the same orientations, while the positions and orientations of the interferers were randomly changed. If any algorithm produced an estimate with a location error greater than $6 \mathrm{~cm}$, it was labeled a "failure" and discarded. Figs. 3 and 4 show the RMS location and orientation errors of the algorithms. The PW-based algorithms have a significantly larger RMS error, even with a substantially larger number of failures removed from the RMS calculation. Figs. 5 and 6 show the RMS 


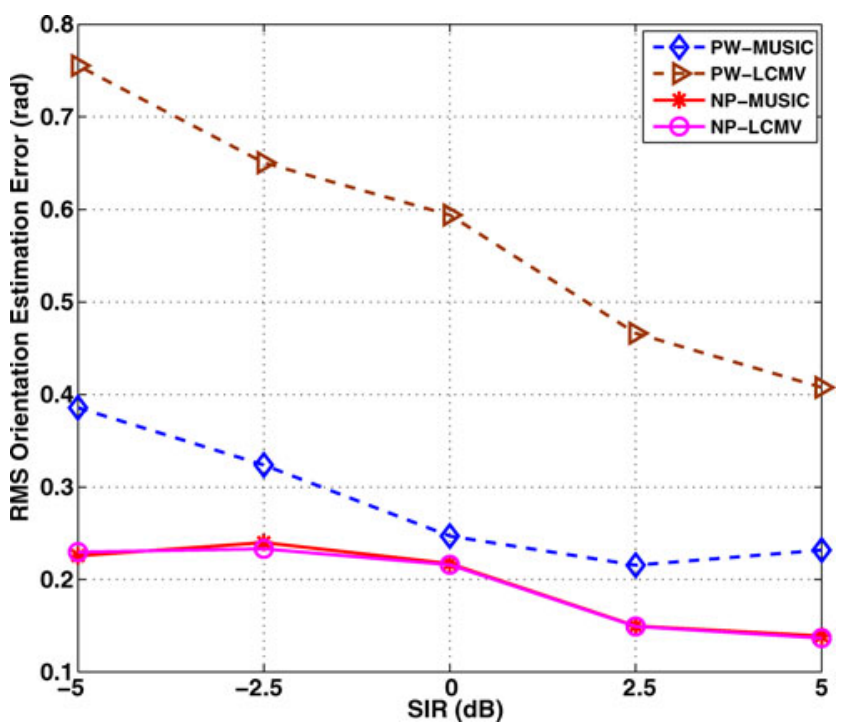

Fig. 4. RMS orientation estimation errors of the four algorithm combinations.

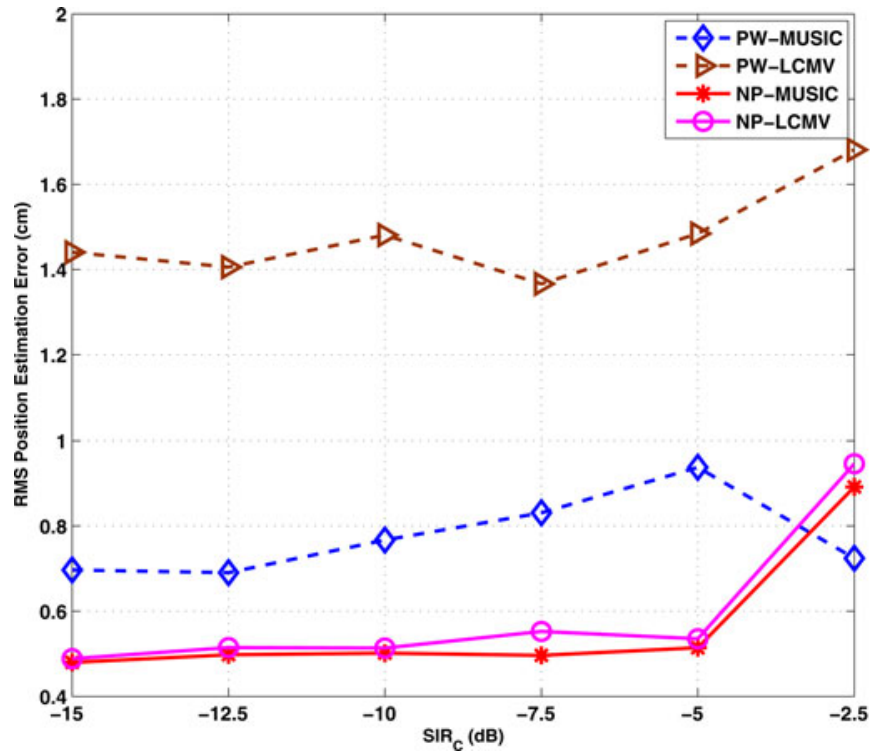

Fig. 5. RMS position estimation errors of the four algorithm combinations in a modulating source scenario.

localization error and failure rate of an experiment conducted to test the impact of the modulating source scenario. The SNR and SIR were set at 15 and $0 \mathrm{~dB}$ with $\operatorname{SIR}_{C}=\left\|\mathbf{S}_{S C}\right\| /\left\|\mathbf{S}_{I C}\right\|$ varying from -15 to $-2.5 \mathrm{~dB}$. As long as $\operatorname{SIR}_{C} \leq-5 \mathrm{~dB}$, or in other words, as long as the stimulus produces at least a $5-\mathrm{dB}$ increase in the strength of the sources of interest, then the NP methods will outperform prewhitening.

Fig. 7 shows the impact of the number of interfering sources on algorithm performance. The SIR was set at $5 \mathrm{~dB}$ in this simulation. As $N_{I}$ increases, the RMS position estimation errors (as well as the orientation errors, although a separate figure is not included here) increase for all of the algorithms. NP still outperforms PW, but the size of the performance gain reduces as $N_{I}$ grows.

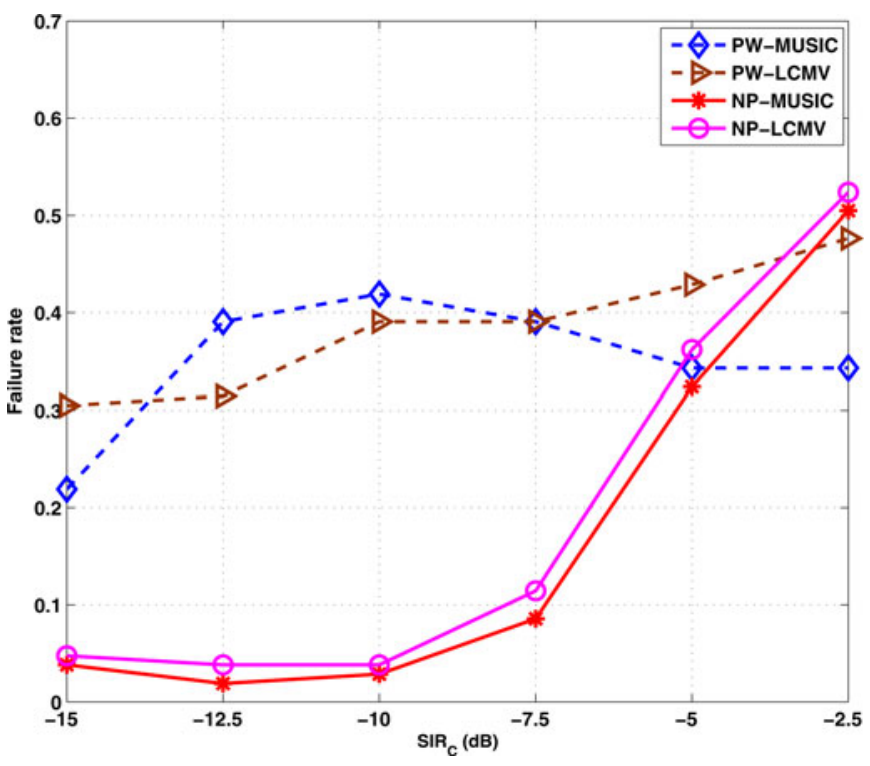

Fig. 6. Failure rate of the four algorithm combinations in the modulating source scenario.

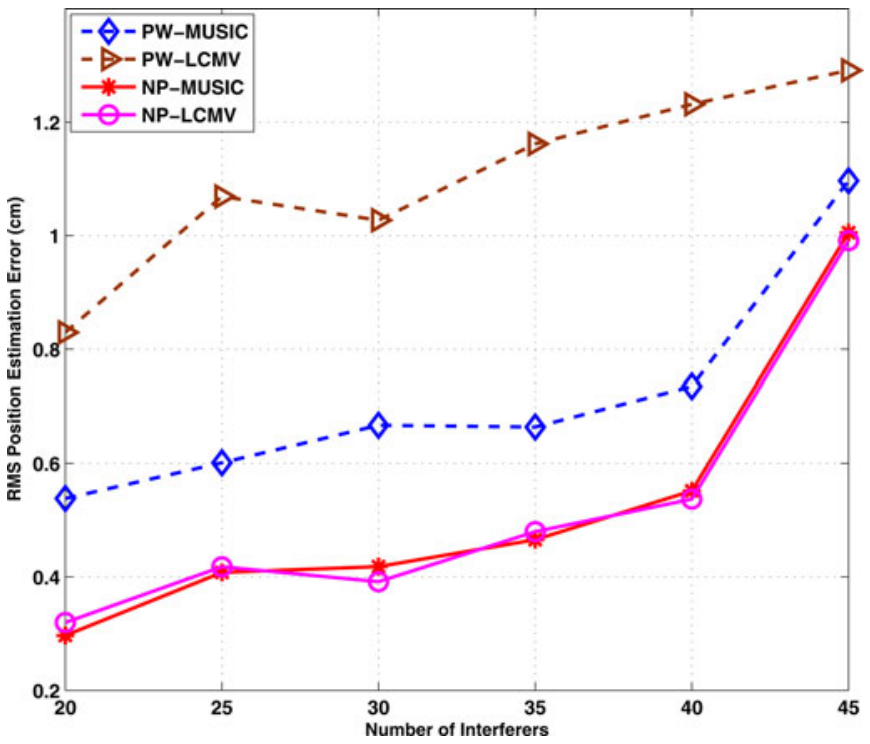

Fig. 7. RMS position estimation errors of the four algorithm combinations under different number of interfering sources.

The final simulation example studies the sensitivity of the algorithms to violations of assumption $\mathrm{A} 4$, which states that the locations of the interfering sources are the same in both the control and activity states. In this example, we set $N_{I}$ and SIR to 25 and $5 \mathrm{~dB}$, respectively, and we perturbed the locations of the interferers in between the control and activity states. In particular, the location of each interfering source during the activity state was moved from its position in the control state a certain distance in a random direction along the brain surface. Fig. 8 shows the RMS position estimation errors as a function of the distance the interferers moved between the two states. While the performance of both NP and PW degrade for large enough source movement, NP retains its performance advantage over PW and shows more resilience than PW to such nonidealities. 


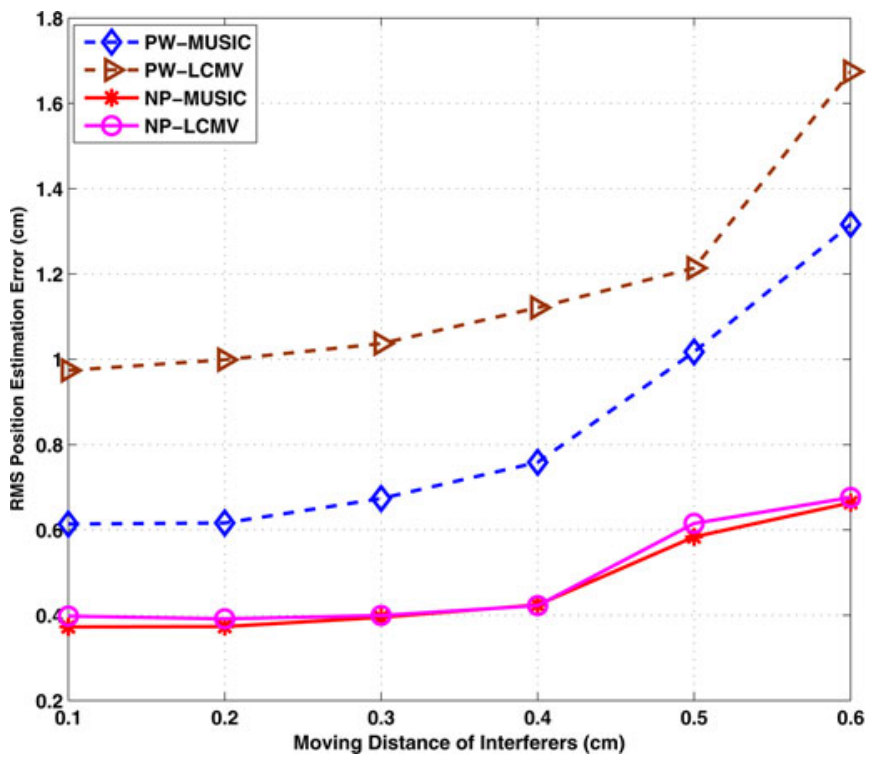

Fig. 8. RMS position estimation errors of the four algorithm combinations when assumption $\mathrm{A} 4$ is violated.

\section{AUDITORY EXPERIMENTS}

Experiments with three different auditory stimuli were conducted with a single human subject to elicit auditory-evoked potentials. The study was approved by the University of California Institutional Review Board and the subject signed an informed consent form. The subject received an auditory stimulus through a pair of stereo (not noise-canceling) headphones and the experiments were conducted in a standard office environment. The subject was not blindfolded. EEG signals were measured using two linked NeXus-32 (MindMedia, RoermondHerten, The Netherlands) systems with linked trigger ports and driven by a synchronization signal so that they were effectively acting as one system. Each NeXus-32 system had a built-in low-pass filter with cutoff frequency at $138 \mathrm{~Hz}$. The stimulus consisted of a 1-kHz pure tone presented to either the subject's left or right ear. Time intervals with no sound were used to generate control state data. The experiment was broken down into 1124 frames, each frame consisting of data recorded with a 512-Hz sampling rate after each possible stimulus event. The length of each frame was randomly varied between 700-800 $\mathrm{ms}$. If the frame contained a stimulus, it occurred during the first $100 \mathrm{~ms}$ and was followed by silence to allow neural activity to die down before the next possible stimulus event. The content of each frame was randomly varied between the two possible stimuli and silence. The outputs of 61 electrodes were recorded, the same as those assumed in the simulations of the previous section. Prior to application of the algorithms described earlier, the data were detrended, the first $700 \mathrm{~ms}$ of each frame was isolated, and then all frames of the same type were averaged together to create the control state and activity state data. The resulting data for the case where the tone was applied to the left ear will be referred to as "Activity L," while "Activity R" will be used to indicate the case where sound was applied to the right ear.

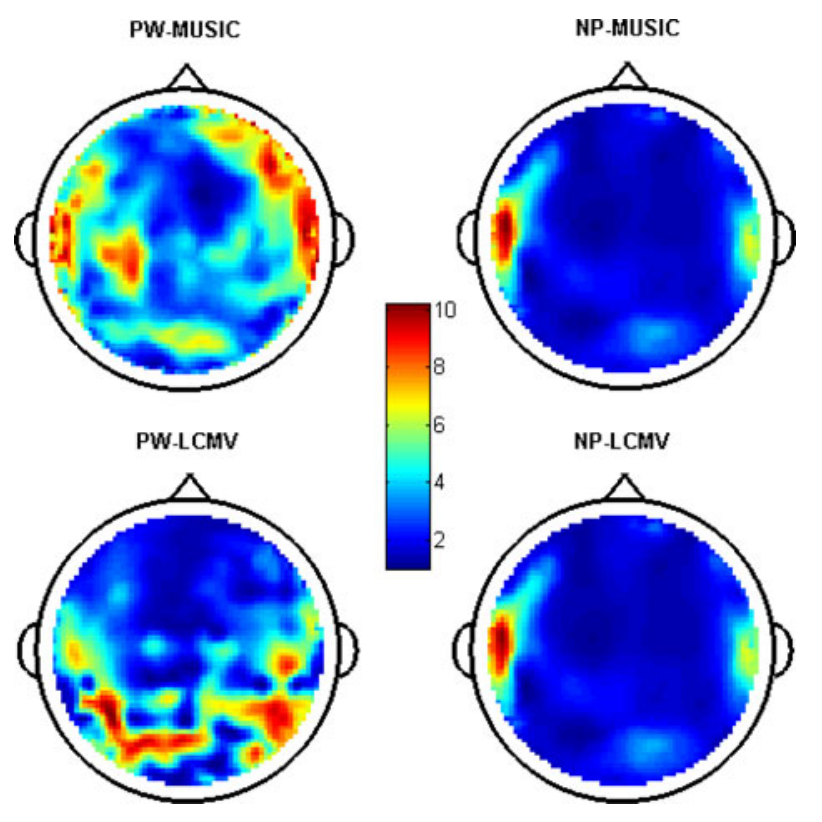

Fig. 9. Spatial spectra of the four algorithm combinations using experimental data for Activity L (top view).

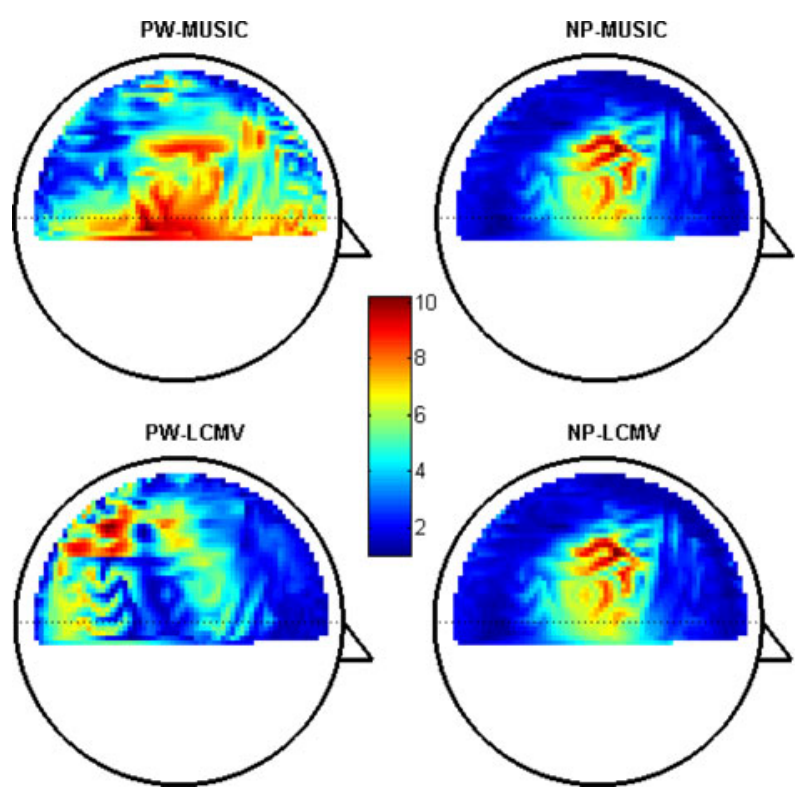

Fig. 10. Same as previous figure (side view).

For auditory-evoked potentials, the P50 and N100 waves are the most relevant to our experiments. They have latencies of about 50 and $100 \mathrm{~ms}$ from stimulus onset and are reported to have a strong contribution from the primary auditory cortex [13], [30]. An SVD of the entire control state data sequence revealed that $99 \%$ of its energy was confined to a rank-19 subspace, and thus, the number of interferers was set to 19 . The actual number of interferers is obviously unknown and could be larger than 19, but for NP only the dimension of the subspace is required. For the activity state data, only samples between 0.04 to $0.2 \mathrm{~s}$ after application of the stimulus were used for localization, in order to isolate the P50 and N100 ERP peaks. MUSIC and LCMV 
were applied to the Activity $\mathrm{L}$ data using both prewhitening and the NP technique and assuming the number of sources was one. Figs. 9 and 10 show the spatial spectra of the four algorithm combinations. Note that the data for these plots have been scaled similarly to those in Figs. 1 and 2 so that the colormaps for all of the figures are consistent. The NP-based methods provide an activity map that closely corresponds to what we expect, with energy confined to the auditory cortex. On the other hand, the PW-based methods contain a number of apparently unrelated artifacts, and the PW-LCMV method does not even show any energy near the auditory cortex. Similar results were obtained for Activity R and are not presented here.

\section{CONCLUSION}

We have presented an efficient deterministic algorithm for the direct suppression of background interference in EEG source localization applications. The proposed method is based on estimating the dominant spatial subspace of the control state data, and then projecting the activity state measurements onto the space orthogonal to it. This approach has the benefit of not requiring any assumptions regarding the temporal stationarity of the interference, and eliminates various difficulties associated with estimating and inverting a large covariance matrix. The performance of the proposed algorithms was demonstrated using both simulations and real data from a simple auditory experiment.

\section{REFERENCES}

[1] J. C. Mosher, P. S. Lewis, and R. M. Leahy, "Multiple dipole modeling and localization from spatio-temporal MEG data," IEEE Trans. Biomed. Eng., vol. 39, no. 6, pp. 541-557, Jun. 1992.

[2] B. D. van Veen, W. van Drongelen, M. Yuchtman, and A. Suzuki, "Localization of brain electrical activity via linearly constrained minimum variance spatial filtering," IEEE Trans. Biomed. Eng., vol. 44, no. 9, pp. 867-880, Sep. 1997.

[3] K. Sekihara, S. S. Nagarajan, D. Poeppel, A. Marantz, and Y. Miyashita, "Application of an MEG eigenspace beamformer to reconstructing spatiotemporal activities of neural sources," Hum. Brain Mapp., vol. 15, pp. 199-215, 2002.

[4] X.-L. Xu, B. Xu, and B. He, "An alternative subspace approach to EEG dipole source localization," Phys. Med. Biol., vol. 49, pp. 327-343, 2004.

[5] Y. S. Chen, C. Y. Cheng, J. C. Hsieh, and L. F. Chen, "Maximum contrast beamformer for electromagnetic mapping of brain activity," IEEE Trans. Biomed. Eng., vol. 53, no. 9, pp. 1765-1774, Sep. 2006.

[6] F. Asano, M. Kimura, D. Shibuya, and Y. Kamitani, "Localization and extraction of brain activity using generalized eigenvalue decomposition," in Proc. IEEE Int. Conf. Acoust., Speech, Signal Process., 2008, pp. 565568.

[7] H. B. Hui, D. Pantazis, S. L. Bressler, and R. M. Leahy, "Identifying true cortical interactions in MEG using the nulling beamformer," NeuroImage, vol. 49, pp. 3161-3174, 2010.

[8] S. C. Wu, A. L. Swindlehurst, P. T. Wang, and Z. Nenadic, "Efficient dipole parameter estimation in EEG systems with near-ML performance," IEEE Trans Biomed Eng., vol. 59, no. 5, pp. 1339-1348, May 2012.

[9] M. Sun, "An efficient algorithm for computing multi-shell spherical volume conductor models in EEG dipole source localization," IEEE Trans. Biomed. Eng., vol. 44, no. 12, pp. 1243-1252, Dec. 1997.

[10] J. Sarvas, "Basic mathematical and electromagnetic concepts of the biomagnetic inverse problem," Phys. Med. Biol., vol. 32, pp. 11-22, 1987.

[11] J. C. Mosher and R. M. Leahy, "Source localization using recursively applied and projected (RAP) MUSIC," IEEE Trans. Signal Processing, vol. 47, no. 2, pp. 332-340, Feb. 1999.

[12] R. Schmidt, "Multiple emitter location and signal parameter estimation," IEEE Trans. Antenn. Propag., vol. 34, no. 3, pp. 276-280, Mar. 1986.
[13] K. Sekihara, K. Hild, and S. Nagarajan, "A novel adaptive beamformer for MEG source reconstruction effective when large background brain activities exist," IEEE Trans. Biomed. Eng., vol. 53, no. 9, pp. 17551764, Sep. 2006

[14] A. Dogandžić, A. Nehorai, "Estimating evoked dipole responses in unknown spatially correlated noise with EEG/MEG arrays," IEEE Trans. Signal Process., vol. 48, no. 1, pp. 13-25, Jan. 2000.

[15] J. C. de Munck, H. M.Huizenga, L. J. Waldorp, and R. A. Heethaar, "Estimating stationary dipoles from MEG/EEG data contaminated with spatially and temporally correlated background noise," IEEE Trans. Signal Process., vol. 50, no. 7, pp. 1565-1572, Jul. 2002.

[16] J. J. Ermer, J. C. Mosher, M. Huang, and R. M. Leahy, "Paired MEG data set source localization using recursively applied and projected (RAP) MUSIC," IEEE Trans. Biomed. Eng., vol. 47, no. 9, pp. 1248-1260, Sep. 2000.

[17] S. Taulua, J. Simola, and M. Kajola, "Presentation of electromagnetic multichannel data: The signal space separation method," IEEE Trans. Signal Process., vol. 97, no. 12, pp. 3359-3372, Jun. 2005.

[18] M. A. Uusitalo and R. J. Ilmoniemi, "Signal-space projection method for separating MEG or EEG into components," Med. Biol. Eng. Comput., vol. 35, pp. 135-140, 1997.

[19] M. Chen, R. T. Wakai, and B. V. Veen, "Eigenvector based spatial filtering of fetal biomagnetic signals," J. Perinatal Med., vol. 29, pp. 486-496, 2001.

[20] P. Parker and A. Swindlehurst, "Space-time autoregressive filtering for matched subspace STAP," IEEE Trans. Aerosp. Electron. Syst., vol. 39, no. 2, pp. 510-520, Apr. 2003.

[21] K. Sekihara, K. Hild, S. S. Dalal, and S. Nagarajan, "Performance of prewhitening beamforming in MEG dual experimental conditions," IEEE Trans Biomed Eng., vol. 55, no. 3, pp. 1112-1121, Mar. 2008.

[22] S. C. Wu, A. L. Swindlehurst, and Y. C. Yao, "Direct interference suppression in EEG/MEG dipole source localization," in Proc. IEEE Int. Conf. Acoust., Speech, Signal Process., 2010, pp. 574-577.

[23] B. N. Cuffin, "EEG localization accuracy improvements using realistically shaped head models," IEEE Trans. Biomed. Eng., vol. 43, no. 3, pp. 299 303, Mar. 1996.

[24] A. Swindlehurst and M. Viberg, "Subspace fitting with diversely polarized antenna arrays," IEEE Trans. Antenn. Propag., vol. 41, no. 12, pp. 16871694, Dec. 1993.

[25] M. Wax and T. Kailath, "Detection of signals by information theoretic criteria," IEEE Trans. Acoust. Speech, Signal Process., vol. 33, no. 2, pp. 387-392, 1985.

[26] X. Bai and B. He, "On the estimation of the number of dipole sources in EEG source localization," Clin. Neurophysiol., vol. 116, pp. 2037-2043, 2005.

[27] R. Oostenveld and P. Praamstra, "The five percent electrode system for high-resolution EEG and ERP measurements," Clin. Neurophysiol., vol. 112, pp. 713-719, 2001.

[28] B. N. Cuffin and D. Cohen, "Comparison of the magnetoencephalogram and electroencephalogram," Electroencephalogr. Clin. Neurophysiol., vol. 47, pp. 132-146, 1979.

[29] S. J. Luck, An Introduction to the Event-Related Potential Technique. Cambridge, MA: MIT Press, 2005.

[30] E. Pekkonen, M. Huotilainen, J. Virtanen, J. Sinkkonen, T. Rinne, R. J. Ilmoniemi, and R. Näätänen, "Age-related functional differences between auditory cortices: A whole-head MEG study," NeuroReport, vol. 6, pp. 1803-1806, 1995.

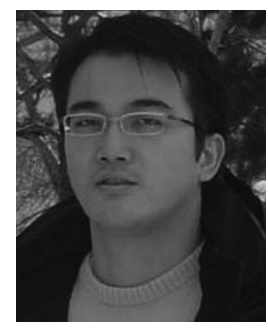

Shun Chi Wu (S'09) received the B.S. and M.S. degrees in engineering and system science both from National Tsing Hua University, Hsinchu, Taiwan, in 2000 and 2002, respectively. He is currently working toward the Ph.D. degree in the Department of Electrical Engineering and Computer Science, University of California, Irvine.

From 2003 to 2007, he was a Research Assistant at the National Space Organization, Hsinchu, Taiwan. His research interests include interference mitigation, feature extraction, source localization/reconstruction, and brain connectivity analysis. 


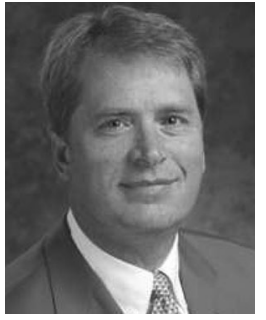

A. Lee Swindlehurst (S'83-M'84-SM'99-F'04) received the B.S. (summa cum laude) and M.S. degrees in electrical engineering both from Brigham Young University, Provo, Utah, in 1985 and 1986, respectively, and the Ph.D. degree in electrical engineering from Stanford University, Stanford, CA, in 1991.

From 1986 to 1990, he was with ESL, Inc., of Sunnyvale, CA, where he was involved in the design of algorithms and architectures for several radar and sonar signal processing systems. From 1990 to 2007, he was with the Faculty of the Department of Electrical and Computer Engineering, Brigham Young University, where he was a Full Professor and served as the Department Chair from 2003 to 2006. During 1996-1997, he held a joint appointment as a Visiting Scholar at both Uppsala University, Uppsala, Sweden, and at the Royal Institute of Technology, Stockholm, Sweden. From 2006 to 2007, he was on leave working as the VicePresident of Research for ArrayComm LLC in San Jose, CA. He is currently a Professor in the Department of Electrical Engineering and Computer Science, University of California, Irvine. His research interests include sensor array signal processing for radar and wireless communications, detection and estimation theory, and system identification, and he has more than 220 publications in these areas.

Dr. Swindlehurst is a past Secretary of the IEEE Signal Processing Society, past Editor-in-Chief of the IEEE Journal OF SELECTED TOPICS IN SIGNAL PROCESSING, and past member of the Editorial Boards for the EURASIP Journal on Wireless Communications and Networking, IEEE Signal Processing Magazine, and the IEEE TRANSACTIONS ON SigNAL PROCESSING. He is a recipient of several paper awards: the 2000 IEEE W. R. G. Baker Prize Paper Award, the 2006 and 2010 IEEE Signal Processing Society's Best Paper Award, the 2006 IEEE Communications Society Stephen O. Rice Prize in the Field of Communication Theory, and is co-author of a paper that received the IEEE Signal Processing Society Young Author Best Paper Award in 2001.

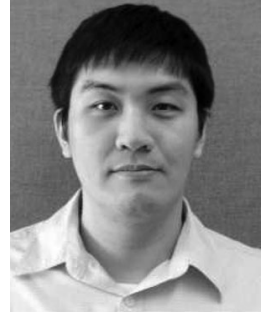

Po T. Wang (S'09) received the B.S. degree in bioengineering from Rice University, Houston, TX, in 2007, and the M.S. degree in biomedical engineering from the University of California, Irvine, in 2008, where he is currently working toward the Ph.D. degree in the Department of Biomedical Engineering.

His research interests include brain-computer interfaces and machine learning.

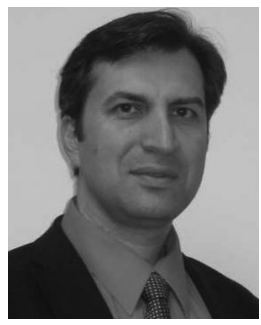

Zoran Nenadic (M'06) received the Diploma degree in control engineering from the University of Belgrade, Belgrade, Serbia, in 1995, and the M.S. and D.Sc. degrees in systems science and mathematics both from Washington University, St. Louis, MO, in 1998 and 2001, respectively.

From 2001 to 2005, he was a Postdoctoral Scholar with the Division of Engineering and Applied Science at the California Institute of Technology, Pasadena. Since 2005, he has been with the Department of Biomedical Engineering, University of California, Irvine, where he is currently an Associate Professor. His research interests include the area of neuroengineering, biomedical signal processing and pattern recognition, biological neural network modeling, and biomedical devices and sensors.

Dr. Nenadic is the recipient of the National Science Foundation Faculty Early Career Development Award. He is a member of the Mathematical Association of America and the Society for Neuroscience. 